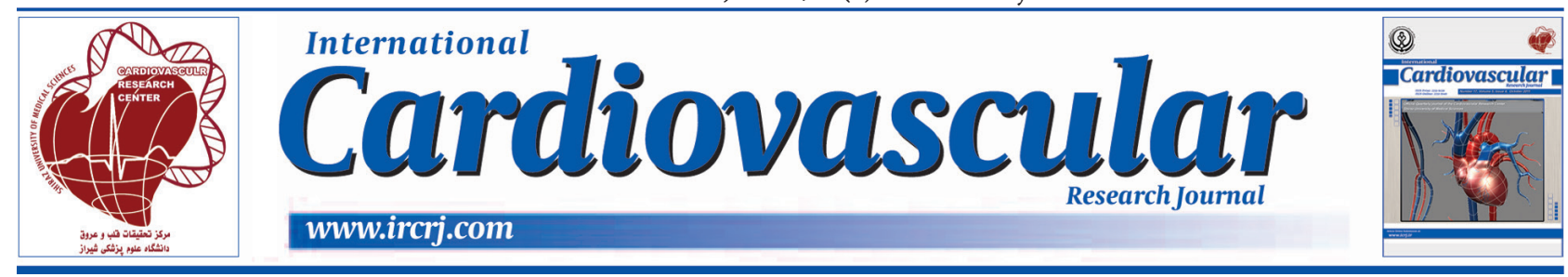

\title{
Tissue Doppler Findings in Patients with Pulmonary Arterial Hypertension
}

\author{
Firoozeh Abtahi ${ }^{1}$, Mohammad Javad Zibaeenezhad ${ }^{1}$, Fatemeh Shafazadeh ${ }^{1}$, Maryam Tahamtan ${ }^{1, *}$
}

${ }^{1}$ Cardiovascular Research Center, Shiraz University of Medical Sciences, Shiraz, IR Iran

\begin{tabular}{l}
\hline A R T I C L E I N F O \\
\hline Article Type: \\
Research Article \\
\hline
\end{tabular}

Article History:

Received: 07 Oct 2015

Revised: 21 Jan 2016

Accepted: 09 Mar 2016

\section{Keywords:}

Pulmonary Arterial Hypertension

Echocardiography

Doppler

Systole

Diastole

\begin{abstract}
A B S T R A C T
Background: Pulmonary hypertension is an untreatable condition with poor prognosis and factors such as more elevated pulmonary arterial systolic pressure and right ventricular dysfunction are associated with a worse outcome.

Objectives: Considering the limitations of the current modalities, this study aimed to find the relationship between tissue Doppler-derived systolic and diastolic parameters and elevated pulmonary arterial pressure in order to assess the routine application of tissue Doppler imaging in evaluation of pulmonary arterial hypertension.

Patients and Methods: This study was conducted on 100 inpatient and outpatient individuals referred to the Department of Echocardiography in Shahid Faghihi hospital, Shiraz, Iran from July 2012 to March 2013. The individuals who had preserved right ventricular function in the presence of pulmonary arterial hypertension were included in the case group. On the other hand, the patients who did not have echocardiographic signs of pulmonary arterial hypertension were enrolled into the control group. All the patients underwent a complete transthoracic echocardiogram including 2-dimensional, color flow, and spectral Doppler as well as tissue Doppler imaging using a vivid E9 system, and the desired systolic and diastolic parameters were recorded. The relationship among these parameters was evaluated by independent sample t-test using the SPSS statistical software, version 16 . Besides, $\mathrm{P}<0.05$ was considered to be statistically significant.

Results: The mean time to peak strain was significantly longer in the case group (203.98 \pm 47.54 milliseconds) compared to the controls ( $81.20 \pm 25.76$ milliseconds). The mean early diastolic tricuspid inflow velocity/early diastolic tricuspid annulus velocity (E/E'a) ratio was also significantly higher in the case group (10.24 \pm 6.19$)$ compared to the controls (4.70 \pm 0.80 ). In contrast, Isovolumic Contraction (IVC) acceleration time/IVC time ratio was reduced in the case group $(0.44 \pm 0.09)$ in comparison to the control patients $(0.56 \pm 0.05)$. Conclusions: In conclusion, our results suggested that increasing degrees of pulmonary artery systolic pressure affected timing of some tissue Doppler-derived intervals within the cardiac cycle, including IVC time, time to peak systolic myocardial velocity (Sm), and time to peak strain. Therefore, tissue Doppler imaging could be used in assessment of patients with suspected pulmonary arterial hypertension.
\end{abstract}

Implication for health policy/practice/research/medical education:

Considering the limitations of the current modalities, this study aimed to find the relationship between tissue Doppler-derived systolic and diastolic parameters and elevated pulmonary arterial pressure in order to assess the routine application of tissue Doppler imaging in evaluation of pulmonary arterial hypertension.

\section{Background}

As a serious cardiopulmonary disease, Pulmonary

${ }^{*}$ Corresponding author: Maryam Tahamtan, Cardiovascular Research Center, Mohammad Rasoul-allah Research Tower, Shiraz University of Medical Sciences, Shiraz, IR Iran. Tel: + 98-7136281561, Fax: +98-7136281562, E-mail:mtahamtan@sums.ac.ir
Hypertension $(\mathrm{PH})$ is an untreatable condition with poor prognosis. According to World Health Organization's (WHO) categorization of PH, Pulmonary Arterial Hypertension (PAH) is one form that is histopathologically defined by presence of vascular proliferation and vasoconstriction 
along with in situ thrombosis (1). Factors, such as more elevated Pulmonary Arterial Systolic Pressure (PASP) and Right Ventricular (RV) dysfunction, are associated with a worse outcome in pulmonary hypertensive patients (24). As a gold standard and direct method of measuring PASP, right heart catheterization cannot be used for regular routine follow-up of patients. Considering such limitations of catheter-based modalities, a reproducible and yet noninvasive technique is required (1-5).

PASP can be estimated through Doppler echocardiographic evaluation of tricuspid regurgitation recordings limited by its dependence on pressure difference among right heart chambers, RV stiffness, and Tricuspid Regurgitation (TR) severity (6). Introduction of Tissue Doppler Imaging (TDI) has provided an invaluable modality in measuring systolic and diastolic time intervals in a single cardiac cycle $(7,8)$. Despite an increasing tendency to use TDI in assessment of cardiac function, usefulness of this modality in assessment of PAH has not been fully established yet.

\section{Objectives}

The present study aims to assess the relationship between TDI-derived systolic and diastolic parameters and elevated pulmonary arterial pressure (PAP), which is the first study on this issue in Iran.

\section{Patients and Methods}

\subsection{Selection of Patients}

The present analytical, cross-sectional study was approved by our institutional review board. The study was conducted on 100 inpatient and outpatient individuals referred to the Department of Echocardiography in Shahid Faghihi hospital, Shiraz, Iran from July 2012 to March 2013. The case group included the patients with preserved RV function documented by Tricuspid Annular Peak Systolic Excursion (TAPSE) values over $16 \mathrm{~mm}$ in the presence of PAH defined by mean PAP of more than $25 \mathrm{mmHg}$ (using TR jet as discussed further in echocardiographic examination section). On the other hand, the control group included the patients who did not have echocardiographic evidence of PAH (neither high-gradient TR nor hypertensive Pulmonary Regurgitation (PR); i.e., mean PAP less than $25 \mathrm{mmHg}$ for both) and were age- and sex-matched with the case group. However, the individuals who had non-sinus rhythms, pacemaker or Implantable Cardioverter-Defibrilator (ICD) leads in RV, or interaventricular conduction delay in electrocardiograms or were unwilling to cooperate were excluded from the study. Based on the similar previous studies and considering power of 90\%, a 100-patient sample size was determined for the study (50 subjects in each group). The patients in both groups were selected via Random Digit Dialing (RDD) method. Additionally, the excluded individuals were substituted by other patients considering the inclusion criteria of the study.

\subsection{Echocardiographic Examination}

All the patients underwent a complete transthoracic echocardiography including 2-dimensional, color flow, and spectral Doppler as well as TDI using a vivid E9 system. It should be mentioned that all the echocardiograms were performed by one cardiologist. The mean PAP was measured by tracing of TR jet on Doppler profile. Besides, PASP was estimated by calculating the systolic pressure gradient between the RV and Right Atrium (RA) by the maximum velocity of the TR jet using the modified Bernoulli equation followed by adding an estimated RA pressure (based on the size of the inferior vena cava and its inspiratory variation according to the guidelines for echocardiographic assessment of the right heart in adults) to both gradients (8). Absence of PAH in the control group was also approved by the mean PAP measured by adding RA pressure to peak early diastolic PR gradient. Tissue Doppler images were captured from the 4 chamber view as routinely performed in our echocardiography laboratory. The region of interest was RV free wall for investigating myocardial systolic and diastolic velocities along with myocardial times. Simultaneously, electrocardiographic documentation of cardiac electrical activity was performed throughout the cardiac cycle for each patient.

\subsection{Statistical Analysis}

At first, normal distribution of the data was confirmed using Kolmogorov-Smirnov test. Therefore, independent sample t-test was used for comparison of the data in the case and control groups. These results were expressed as mean \pm Standard Deviation (SD). All data analyses were performed by the SPSS statistical software, version 16.0 (Chicago, IL) and P values less than 0.05 were considered to be statistically significant.

\section{Results}

The mean age of the participants was 49 years, ranging from 30 to 60 years. In addition, the mean age of the patients in the case and control groups was 50.5 (33 - 60) and 49.3 years (31 - 60), respectively. Besides, both groups showed similar gender distribution and half of the patients in each group were male. Therefore, no statistically significant difference was observed between pulmonary hypertensive and control groups regarding age and gender.

The mean PAP was $53 \mathrm{mmHg}$ and the mean PASP was $78 \mathrm{mmHg}$ in the case group. On the other hand, the mean PAP was 11.6 in the control group. Furthermore, the mean TAPSE was $18 \mathrm{~mm}$ in the cases and $20 \mathrm{~mm}$ in the controls. The mean pulmonary acceleration time was also $63.72 \pm$ 11.06 milliseconds (msec) in the pulmonary hypertensive patients and $139.00 \pm 17.54 \mathrm{msec}$ in the controls $(\mathrm{P}<0.001)$.

The mean Isovolumic Contraction Acceleration (IVC acc) time and time to peak systolic myocardial velocity (time to peak Sm) were higher in the control individuals compared to the cases $(\mathrm{P}<0.001,95 \% \mathrm{CI})$. The results revealed a statistically significant difference between the case and control groups regarding the mean of IVC time ( $\mathrm{P}$ $<0.001)$. Accordingly, the duration of IVC was shorter in the pulmonary hypertensive individuals $(64.2 \pm 19.83 \mathrm{msec})$ compared to the controls $(82.6 \pm 7.81 \mathrm{msec})$. The results also showed a significant difference between the two groups with respect to IVC acc time/IVC time $(\mathrm{P}<0.001)$. Based on the results, this ratio was reduced in the case individuals $(0.44$ $\pm 0.09)$ in comparison to the controls $(0.56 \pm 0.057)$. On the other hand, the mean peak systolic myocardial velocity 
(Sm), early diastolic tricuspid inflow velocity (E), and tissue Doppler-derived early diastolic tricuspid annular velocity (E'a) were substantially higher in the control patients compared to the case group. Nonetheless, the mean early diastolic tricuspid inflow velocity/early diastolic tricuspid annular velocity (E/E'a) ratio was significantly higher in the case group (10.24 \pm 6.19$)$ in comparison to the controls (4.70 $\pm 0.80)$. The mean time to peak strain was also significantly longer in the cases compared to the controls $(203.98 \pm 47.54$ vs. $81.20 \pm 25.76 \mathrm{msec}, \mathrm{P}<0.001)$. All the measured systolic and diastolic parameters have been summarized in Table 1. Besides, a sample of the TDI profile of the RV parameters has been demonstrated in Figures 1 and 2 .

\section{Discussion}

The present study aimed to assess the relationship between tissue Doppler-derived systolic and diastolic parameters and elevated pulmonary arterial pressure in an Iranian population. According to the results, the mean time to peak strain was significantly longer in the case individuals in comparison to the controls. These findings are comparable to those reported in the study by Lopez where pulmonary hypertensive patients had a significantly delayed time-topeak strain between the interventricular septum and the RV free wall $(66 \pm 59 \mathrm{~ms})$ compared to the controls $(8 \pm$ $11 \mathrm{~ms}, \mathrm{P}<0.0001$ ) (9). Lopez et al. also conducted a study revealing significantly diminished tricuspid annular peak systolic velocity in patients with PAH compared to normal individuals, which is in agreement with our study results. It should be mentioned that the sample size of both studies were similar in the case and control groups $(n=50)(10)$.

Other studies have also proved the usefulness of tricuspid annular TDI in evaluation of RV systolic and diastolic functions (11-14). It is noteworthy that annular TDI is not only less technically demanding than either RV fractional area change calculation or M-mode measurement of maximal annular systolic excursion (TAPSE), but is also a reliable method for detecting changes as a result of $\mathrm{PAH}$, as shown in the current study. Therefore, factors, such as ease of acquisition, reliability, and technical capability of TDI, make tricuspid annular TDI a provocative imaging tool that has never been the subject of studies evaluating the biventricular effects of PH. Yet, several studies have combined tissue Doppler parameters of RV function with

\begin{tabular}{|c|c|c|c|}
\hline & $\operatorname{PAH}^{\mathrm{a}}(\mathrm{N}=50)$ & Control $(\mathrm{N}=50)$ & P value \\
\hline Pulmonary acceleration time (msec) & $63.72 \pm 11.06$ & $139.00 \pm 17.54$ & $<0.001$ \\
\hline Isovolumic contraction acceleration time (msec) & $28.52 \pm 9.73$ & $46.96 \pm 6.31$ & $<0.001$ \\
\hline Isovolumic contraction time (msec) & $64.20 \pm 19.83$ & $82.60 \pm 7.81$ & $<0.001$ \\
\hline IVC $^{b}$ acceleration time/IVC time & $0.44 \pm 0.09$ & $0.56 \pm 0.057$ & $<0.001$ \\
\hline Time to peak Sm & $126.32 \pm 27.97$ & $188.48 \pm 20.1$ & $<0.001$ \\
\hline $\operatorname{Peak} \mathrm{Sm}^{\mathrm{c}}(\mathrm{m} / \mathrm{s})^{\mathrm{d}}$ & $0.10 \pm 0.02$ & $0.12 \pm 0.01$ & $<0.001$ \\
\hline Transtricuspid early diastolic velocity (E) (m/s) & $0.70 \pm 0.18$ & $0.51 \pm 0.05$ & $<0.001$ \\
\hline Lateral tricuspid annular velocity during early diastole (E’a) (m/s) & $0.08 \pm 0.35$ & $0.11 \pm 0.01$ & $<0.001$ \\
\hline Transtricuspid to lateral tricuspid annlar early diastolic velocity ratio (E/Ea) & $10.24 \pm 6.19$ & $4.70 \pm 0.80$ & $<0.001$ \\
\hline Time to peak strain $(\mathrm{msec})^{\mathrm{e}}$ & $203.98 \pm 47.54$ & $81.20 \pm 25.76$ & $<0.001$ \\
\hline
\end{tabular}

Abbreviations: ${ }^{\text {a }} \mathrm{PAH}$, pulmonary arterial hypertension; ${ }^{\mathrm{b}} \mathrm{IVC}$, isovolumic contraction; ${ }^{\mathrm{c}} \mathrm{Sm}$, systolic myocardial velocity; ${ }^{\mathrm{d}} \mathrm{m} / \mathrm{s}$, meters per second; ${ }^{\mathrm{e}} \mathrm{msec}$, millisecond

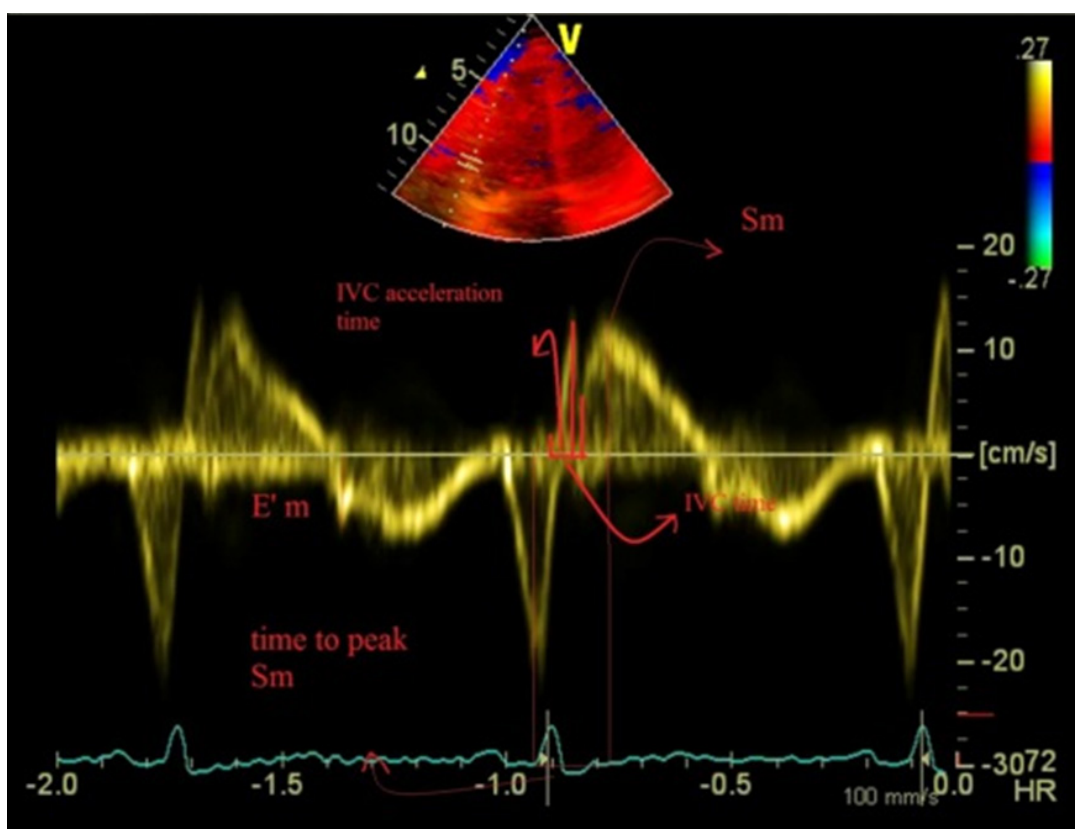

Figure 1. Tissue Doppler Profile of the Right Ventricle Representing Measurement of IVC Time, IVC Acceleration Time, Sm, Time to Peak Sm, and E'a Parameters 


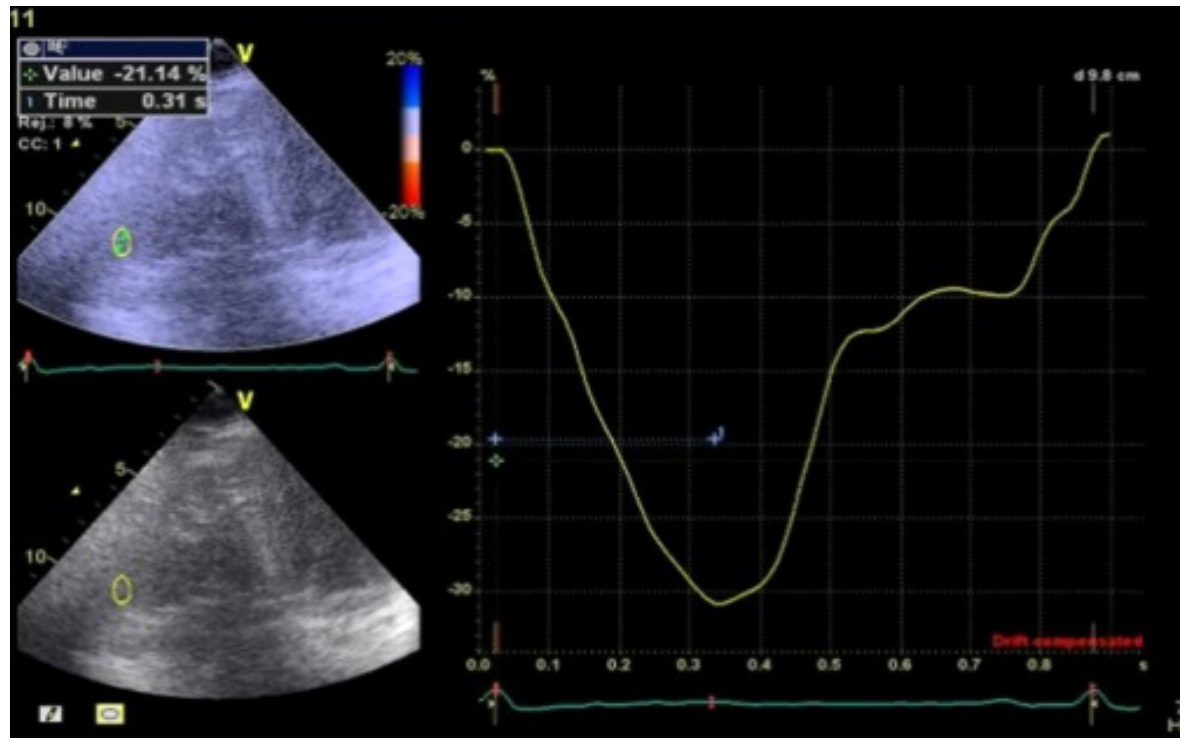

Figure 2. Tissue Doppler Imaging of the Right Ventricle, Revealing the Measurement of Time to Peak Strain

other factors, such as 3-dimensional factors. Such studies are superior to ours since they provide sensitivity, specificity, and positive and negative predictive values in detecting PAH. For instance, Stefano De Castro et al. compared an easily-measured parameter, peak systolic velocity of tricuspid annulus (TAPSV) obtained by TDI, to RV Ejection Fraction (RVEF) measured by real time 3-dimensional echocardiography. They also aimed to explore whether TAPSV cutoff values would be useful in detecting global RV dysfunction. The study results indicated a statistically significant correlation between TAPSV and RVEF ( $\mathrm{P}<$ 0.001). Moreover, with RV dysfunction defined as RVEF $<40 \%$, a TAPSV cutoff value of $9.5 \mathrm{~cm} / \mathrm{sec}$ yielded significantly high sensitivity and specificity and appeared to be a valid compromise in detecting RV dysfunction (15).

Melek et al. assessed the relationship between tricuspid lateral annulus TDI parameters and PASP estimated by continuous wave Doppler. They studied 51 patients with clinically stable chronic obstructive pulmonary disease. The Sm, velocity time integral of Sm (SmVTI), E'a, tissue Doppler-derived late diastolic tricuspid annular velocity (A'a), myocardial Isovolumic Relaxation Time (IVRT), and 2-dimensional and conventional Doppler data were acquired. The results demonstrated that in comparison to the patients without PH, Sm, SmVTI, E'a, and E'a/A'a were lower and IVRT was higher in those with PH. These findings are in agreement with those of our study, which investigated the same issue in an Iranian population. In conclusion, they suggested that in cases where noninvasive PASP measurement is not possible, TDI can be used as an alternative and reliable method to assess PAP (16).

One of the limitations of this study was its small sample size and lack of outcome data, which curtailed our ability to draw definitive conclusions. Besides, we lacked the sequential imaging of the patients in the early stages of the disease that would have been ideal to validate the true utility of these TDI parameters with worsening degrees of PAH. Moreover, we did not analyze the effect of different loading conditions on RV myocardial velocity and strain. Finally, since this study was the first one in Iranian population, the results should be confirmed by further similar studies worldwide enrolling a larger number of subjects.

\subsection{Conclusion}

In conclusion, our results suggested that increasing degrees of PASP affected timing of some TDI-derived intervals within the cardiac cycle, including IVC time, time to peak Sm, and time to peak strain. Yet, these findings should be confirmed in further longitudinal studies with larger sample sizes. Future studies are also required to assess the sufficient sensitivity of these intervals for routine follow-up examination to detect changes over time or with treatment of PAH. Furthermore, RV free wall strain appeared to be useful in identifying abnormal RV deformation in PAH and should be further assessed as a potential mechanism of RV change in the setting of chronic pressure overload.

\section{Acknowledgements}

The study was supported by Shiraz Cardiovascular Research Center.

\section{Authors' Contribution}

Firoozeh Abtahi and Mohammad Javad Zibaeenezhad: Design, Fatemeh Shafazadeh: Data collection and analysis, Maryam Tahamtan: Data collection and report of the results

\section{Financial disclosure}

There is no financial disclosure.

\section{Funding/Support}

There is no funding/support.

\section{References}

1. Schannwell CM, Steiner S, Strauer BE. Diagnostics in pulmonary hypertension. J Physiol Pharmacol. 2007;58 Suppl 5(Pt 2):591-602.

2. Gavazzi A, Ghio S, Scelsi L, Campana C, Klersy C, Serio A, et al. Response of the right ventricle to acute pulmonary vasodilation predicts the outcome in patients with advanced heart failure and pulmonary hypertension. Am Heart J. 2003;145(2):310-6.

3. Noly PE, Guihaire J, Coblence M, Dorfmuller P, Fadel E, Mercier O. Chronic Thromboembolic Pulmonary Hypertension and Assessment of Right Ventricular Function in the Piglet. J Vis Exp. 
2015(105):e53133.

4. Raymond RJ, Hinderliter AL, Willis PW, Ralph D, Caldwell EJ, Williams W, et al. Echocardiographic predictors of adverse outcomes in primary pulmonary hypertension. $\mathrm{J} \mathrm{Am} \mathrm{Coll} \mathrm{Cardiol.}$ 2002;39(7):1214-9.

5. Oudiz RJ, Ginzton L. Pulmonary artery systolic pressure estimated by echocardiogram vs catheterization in patients awaiting lung transplantation. J Heart Lung Transplant. 2003;22(7):832-3; author reply 3 .

6. Homma A, Anzueto A, Peters JI, Susanto I, Sako E, Zabalgoitia M, et al. Pulmonary artery systolic pressures estimated by echocardiogram vs cardiac catheterization in patients awaiting lung transplantation. J Heart Lung Transplant. 2001;20(8):833-9.

7. Harada K, Tamura M, Toyono M, Yasuoka K. Comparison of the right ventricular Tei index by tissue Doppler imaging to that obtained by pulsed Doppler in children without heart disease. Am J Cardiol. 2002;90(5):566-9.

8. Rudski LG, Lai WW, Afilalo J, Hua L, Handschumacher MD, Chandrasekaran K, et al. Guidelines for the echocardiographic assessment of the right heart in adults: a report from the American Society of Echocardiography endorsed by the European Association of Echocardiography, a registered branch of the European Society of Cardiology, and the Canadian Society of Echocardiography. $J$ Am Soc Echocardiogr. 2010;23(7):685-713; quiz 86-8.

9. Lopez-Candales A, Rajagopalan N, Dohi K, Edelman K, Gulyasy B. Normal range of mechanical variables in pulmonary hypertension: a tissue Doppler imaging study. Echocardiography. 2008;25(8):864-72.
10. Lopez-Candales A, Edelman K, Gulyasy B, Candales MD. New annular tissue Doppler markers of pulmonary hypertension. Echocardiography. 2010;27(8):969-76.

11. Bigdelu L, Azari A, Fazlinezhad A. Assessment of Right Ventricular Function by Tissue Doppler, Strain and Strain Rate Imaging in Patients with Left-Sided Valvular Heart Disease and Pulmonary Hypertension. Archives of Cardiovascular Imaging. 2014;2(1).

12. Kjaergaard J. Assessment of right ventricular systolic function by tissue Doppler echocardiography. Dan Med J. 2012;59(3):B4409.

13. Rajagopalan N, Saxena N, Simon MA, Edelman K, Mathier MA, Lopez-Candales A. Correlation of tricuspid annular velocities with invasive hemodynamics in pulmonary hypertension. Congest Heart Fail. 2007;13(4):200-4.

14. Sade LE, Gulmez O, Eroglu S, Sezgin A, Muderrisoglu H. Noninvasive estimation of right ventricular filling pressure by ratio of early tricuspid inflow to annular diastolic velocity in patients with and without recent cardiac surgery. $J$ Am Soc Echocardiogr. 2007;20(8):982-8.

15. De Castro S, Cavarretta E, Milan A, Caselli S, Di Angelantonio E, Vizza Carmine D, et al. Usefulness of tricuspid annular velocity in identifying global RV dysfunction in patients with primary pulmonary hypertension: a comparison with 3D echo-derived right ventricular ejection fraction. Echocardiography. 2008;25(3):289-93.

16. Melek M, Esen O, Esen AM, Barutcu I, Fidan F, Onrat E, et al. Tissue Doppler evaluation of tricuspid annulus for estimation of pulmonary artery pressure in patients with COPD. Lung. 2006;184(3):121-31. 